\title{
PLANO DE PARTO COMO INSTRUMENTO DAS BOAS PRÁTICAS NO PARTO E NASCIMENTO: REVISÃO INTEGRATIVA
}

\author{
BIRTH PLAN AS AN INSTRUMENT OF GOOD \\ PRACTICES IN DELIVERY AND BIRTH: \\ AN INTEGRATIVE REVIEW
}

\section{PLAN DE PARTO COMO INSTRUMENTO DE BUENAS PRÁCTICAS EN EL TRABAJO DE PARTO Y EN EL PARTO: UNA REVISIÓN INTEGRADORA}

\author{
Wanessa Nathally de Santana Silva ${ }^{1}$ \\ Joicy Amorim Francisco de Azevêdo ${ }^{1}$ \\ Viviane Rolim de Holanda ${ }^{2}$ \\ Ana Lisa do Vale Gomes ${ }^{3}$ \\ Geyslane Pereira Melo de Albuquerque ${ }^{4}$
}

Como citar este artigo: Silva WNS, Azevedo JAF, Holanda VR, Gomes ALV, Albuquerque GPM. Plano de parto como instrumento das boas práticas no parto e nascimento: revisão integrativa. Rev baiana enferm. 2019;33:e32894.

\begin{abstract}
Objetivo: analisar as evidências disponíveis sobre as principais contribuições do plano de parto como instrumento das boas práticas obstétricas no processo de parto e nascimento em ambiente hospitalar. Método: revisão integrativa com 12 artigos publicados entre 2013 e 2018 nas bases de dados MEDLINE, Biblioteca Virtual de Saúde, LILACS, SciELO e PubMed, utilizando trabalhos originais, reflexão, atualização e relato de experiência, completos e disponíveis na íntegra. Resultados: observou-se a importância do plano de parto para estimular as boas práticas obstétricas, permitir maior autonomia e liberdade de escolha das mulheres, contribuir para um atendimento qualificado e humanizado, facilitar a confiança da mulher com a equipe, além de favorecer maior satisfação com o parto, comunicação com os profissionais envolvidos e propiciar resultados maternos/neonatais mais satisfatórios. Conclusão: comprovou-se que o plano de parto é um instrumento que propicia boas práticas obstétricas no processo de parto e nascimento em ambiente hospitalar.
\end{abstract}

Descritores: Enfermagem. Parto Humanizado. Saúde Materno-infantil.

Objective: to analyze the available evidence on the main contributions of the birth plan as an instrument of good obstetric practices in the process of delivery and birth in a hospital environment. Method: an integrative review with 12 articles published between 2013 and 2018 in the MEDLINE, Virtual Health Library, LILACS, SciELO and PubMed databases, using full and complete original works, reflection, update and experience report. Results: the importance of the birth plan importance was observed to encourage good obstetric practices, allow greater autonomy

\footnotetext{
Enfermeira. Pesquisadora independente. Vitória de Santo Antão, Pernambuco, Brasil. wanessa.nataly@hotmail.com. https://orcid.org/0000-0002-4234-565I; https://orcid.org/0000-0003-2929-7074.

2 Enfermeira. Doutora em Enfermagem. Professora da Universidade Federal da Paraíba. Paraíba, João Pessoa, Brasil. https://orcid.org/0000-0002-72। 2-I800.

Biomédica. Doutora em Ciências. Professora da Universidade Federal de Pernambuco. Vitória de Santo Antão, Pernambuco, Brasil. https://orcid.org/0000-000 I7067-|48|.

4 Enfermeira. Doutora em Enfermagem. Professora Substituta na Universidade Federal de Pernambuco. Vitória de Santo Antão, Pernambuco, Brasil. https://orcid. org/0000-000 I-7246-883।.
} 
and freedom of women's choice, contribute to a qualified and humanized care, facilitate women's trust with the team, and promote greater satisfaction with childbirth, communication with the involved professionals and provide more satisfactory maternal/neonatal results. Conclusion: it was proved that the birth plan is an instrument that provides good obstetric practices in the process of delivery and birth in a hospital environment.

Descriptors: Nursing. Humanized Birth. Maternal-Child Health.

Objetivo: analizar las evidencias disponibles sobre las principales contribuciones del plan de parto como instrumento de las buenas prácticas obstétricas en el proceso de parto y nacimiento en el entorno de un hospital. Método: revisión integradora con 12 artículos publicados entre 2013 y 2018 en las siguientes bases de datos: MEDLINE, Biblioteca Virtual de Saúde, LILACS, SciELO y PubMed, utilizando trabajos originales, de reflexión, de actualización y reporte de experiencias, completos y disponibles en forma íntegra. Resultados: se observó la importancia del plan de parto para estimular las buenas prácticas obstétricas, permitir mayor autonomía y libertad de elección de las mujeres, contribuir a una asistencia calificada y bumanizada, facilitar que la mujer confíe en el equipo médico, además de favorecer una mayor satisfacción con el parto, la comunicación con los profesionales participantes, y propiciar resultados maternos/neonatales más satisfactorios. Conclusión: se comprobó que el plan de parto es un instrumento que propicia buenas prácticas obstétricas en el proceso de parto y nacimiento en un ambiente de hospital.

Descriptores: Enfermería. Parto Humanizado. Salud Materno-Infantil.

\section{Introdução}

A construção do Plano de Parto (PP) geralmente acontece durante o pré-natal. Sua aplicação permite que os profissionais da assistência e as gestantes apropriem-se previamente dos procedimentos que elas gostariam que ocorressem durante o nascimento do seu filho. Dessa forma, permite que os profissionais da assistência respeitem as decisões da mulher e garantam uma assistência de acordo com suas vontades, contribuindo para o empoderamento feminino e a promoção da saúde materna e neonatal ${ }^{(1)}$. O uso do PP é recomendado pela Organização Mundial da Saúde (OMS) e pelo Ministério da Saúde no Brasil após a implantação do Programa Rede Cegonha, em 2011. No PP, as gestantes expressam os cuidados que desejam durante o seu trabalho de parto, parto e pós-parto, que incluem desde a ingesta alimentar e hídrica, à posição na hora de parir, ao uso de analgesia, além de evitar a realização de procedimentos rotineiros sem embasamento científico ${ }^{(2)}$.

O PP é um instrumento educativo, de caráter legal, desenvolvido pelo profissional da assistência, em geral o enfermeiro, durante o período gestacional e apresentado pelas gestantes antes do parto, o que favorece a reflexão e auxilia na sua tomada de decisões sobre o parto e os procedimentos realizados. Além disso, contribui para orientação dos profissionais da assistência em relação ao serviço prestado ${ }^{(3)}$. Este instrumento foi adotado pela primeira vez pelo serviço de saúde da Espanha em 2008. O principal objetivo era garantir que as mulheres expressassem seus desejos e expectativas relacionadas ao trabalho de parto, parto e nascimento. Sua implementação tem sido discutida no contexto clínico e em diversas literaturas ${ }^{(4)}$.

O parto é um momento único na vida da mulher e de seus familiares e deve envolver o processo de humanização durante toda sua trajetória. A garantia de uma assistência humanizada é primordial para prevenir a violência institucional contra as mulheres ${ }^{(5)}$. A humanização no parto começou a ser discutida a partir da década de 1980, quando se percebeu a necessidade de garantir boas práticas durante o parto, devido ao aumento da medicalização e intervenções desnecessárias ${ }^{(5)}$. No Brasil, os cuidados à mulher durante o parto vêm sofrendo diversas modificações ao longo dos anos, tendo como principal objetivo a transformação do modelo intervencionista em uma assistência humanizada, baseada em evidências científicas, que resgatam o poder de escolha e o protagonismo da mulher ${ }^{(6)}$.

Um dos aspectos mais observados durante as práticas obstétricas é a falta de respeito à autonomia das gestantes. Por vezes, percebe-se que a assistência não se baseia na dinâmica do 
corpo da mulher, implicando em intervenções desnecessárias e ocasionando uma experiência de parto negativa ${ }^{(7)}$. Estimular a participação das gestantes no cuidar, durante o período de gestação, parto e nascimento, é uma estratégia importante que deve ser colocada em prática, para se alcançar melhores resultados maternos e perinatais. Nesse contexto, o acesso à informação possibilita maior autonomia da mulher e o PP pode auxiliar no seu empoderamento ${ }^{(8)}$.

Frente a esse contexto, a presente revisão situa-se no campo de estudos que abrange a humanização da assistência no período gestacional, processo de parto e nascimento. De forma mais específica, procura refletir sobre a utilização do $\mathrm{PP}$, com enfoque na sua importância e nos benefícios para as gestantes.

O estudo justifica-se pela necessidade de identificar os achados expressos na literatura científica envolvendo a temática do PP que possam contemplar de forma positiva a sistematização das evidências que fortalecem esses instrumentos como empoderadores das gestantes, da equipe de saúde e dos índices de saúde da mulher e da criança. A maior divulgação do PP, além de acarretar melhorias para a mulher, o recém-nascido e os acompanhantes, traduz-se em um forte indicador de benefícios para a saúde pública. Logo, objetivou-se analisar as evidências disponíveis sobre as principais contribuições do PP como instrumento das boas práticas obstétricas no processo de parto e nascimento em ambiente hospitalar.

\section{Método}

Trata-se de uma revisão integrativa, desenvolvida nas seguintes etapas: elaboração da questão de pesquisa; elaboração dos critérios de elegibilidade e busca na literatura; coleta de dados dos artigos selecionados; análise crítica dos estudos incluídos; discussão dos resultados; apresentação final do artigo de revisão integrativa ${ }^{(9)}$.

Para atingir o objetivo proposto, procurou-se responder a seguinte questão: Quais as contribuições do PP para o cuidado no período do parto e nascimento de acordo com as publicações científicas de enfermagem e da saúde?
A seleção ocorreu por meio do metabuscador da Coordenação de Aperfeiçoamento de Pessoal de Nível Superior (CAPES). Os artigos científicos foram extraídos das fontes eletrônicas: Medical Literature Analysis and Retrieval System Online/ Biblioteca Virtual em Saúde (MEDLINE/BVS), Literatura Latino-Americana e do Caribe em Ciências da Saúde (LILACS), PubMed Central (PMC), e Scientific Electronic Library Online (SciELO).

Foram incluídos artigos científicos internacionais e nacionais, publicados entre 2013 e 2018, nas categorias de original, revisão de literatura, reflexão, atualização, relato de experiência e textos completos, disponíveis na íntegra ou obtidos por meio do Programa de Comutação Bibliográfica (COMUT) da Universidade Federal de Pernambuco (UFPE). Excluíram-se estudos do tipo teses e dissertações, livros, trabalhos de conclusão de curso, resumos, revisões e cartilhas.

Para a busca criteriosa dos artigos, utilizaram-se os descritores - trabalho de parto, parto humanizado, parto, tomada de decisões - e seus respectivos correlatos nos idiomas inglês e espanhol em cada base de dados, consultados nos Descritores em Ciências da Saúde (DeCS) e no Medical Subject Headings (MeSH). Para apreciação da produção científica relacionada ao tema proposto, três pesquisadoras independentes avaliaram os registros. A estratégia de busca dos estudos primários pelos descritores nas bases de dados foi sistematizada e combinada de diferentes formas, cruzadas com o operador lógico booleano "AND" com combinações em pares e tríades, permitindo, assim, a busca ampla dos estudos primários: "Trabalho de parto" AND "Tomada de decisões"; "Parto" AND "Tomada de decisões"; "Parto humanizado" AND "Tomada de decisões"; "Parto humanizado" AND "Trabalho de parto" AND "Tomada de decisões"; "Parto humanizado" AND "Parto" AND "Tomada de decisões".

As estratégias de busca ocorreram entre maio e dezembro de 2018. Foram extraídas as principais informações de cada documento selecionado, de acordo com um instrumento adaptado: identificação (título da publicação, autor principal, idioma e ano de publicação), objetivo e 
tipo de estudo, nível de evidência, avaliação dos resultados encontrados ${ }^{(10)}$.

Nas bases de dados pesquisadas, foram encontrados 829 estudos. Os itens duplicados foram descartados ${ }^{(751)}$. Dos 78 artigos restantes, revisaram-se os títulos e os resumos conforme seu conteúdo. Destes, 25 resumos preencheram os critérios de seleção e foram submetidos a uma revisão completa do texto. Após leitura na íntegra, foram selecionados 12 artigos, conforme apresentado na Figura 1. Por fim, esses artigos de boa qualidade metodológica foram classificados quanto ao nível de evidência proposto por Melnyk $^{(11)}$.

Fluxograma 1 - Seleção dos artigos

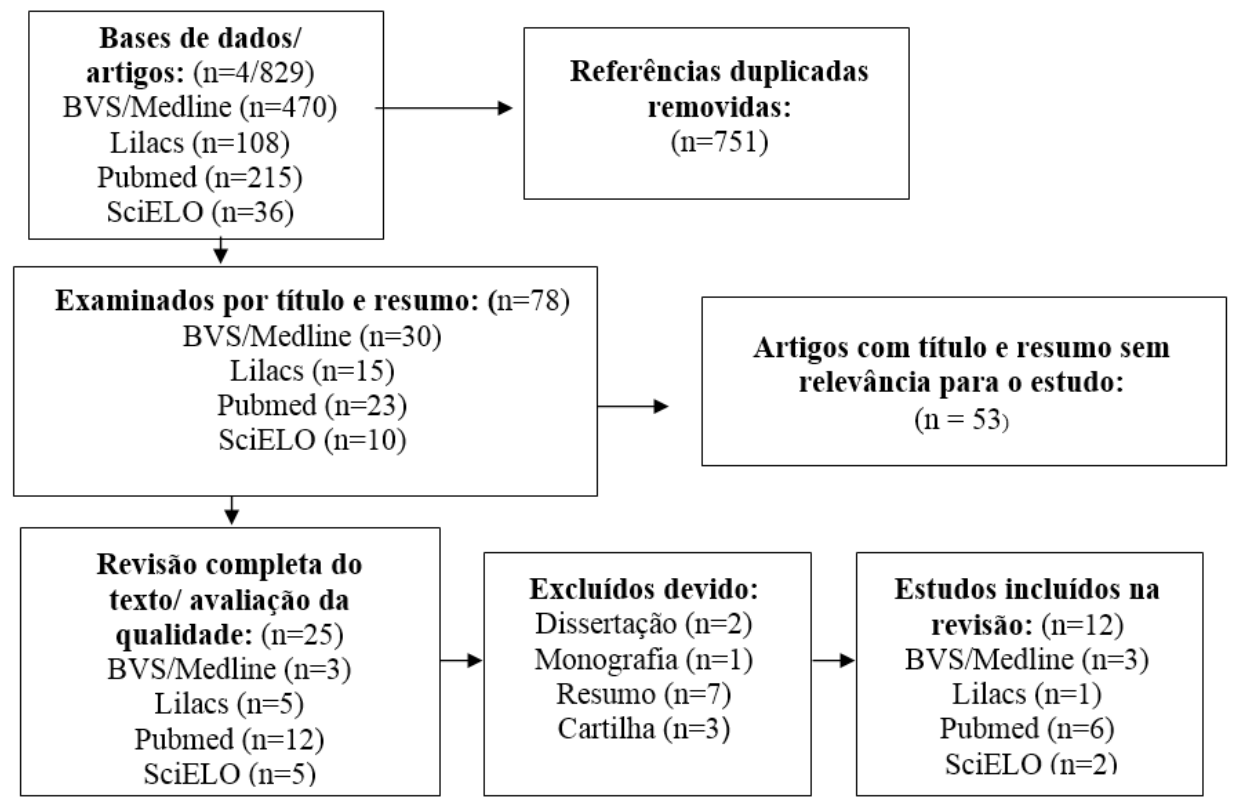

Fonte: Elaboração própria.

\section{Resultados}

Com relação ao idioma, observou-se que cinco artigos foram publicados em português, quatro em espanhol e três em inglês. De maneira geral, a temática envolvendo o PP tem sido discutida de forma benéfica na literatura da saúde e tem contribuído positivamente para os cuidados antes, durante e depois do parto. No Quadro 1 são descritas as características dos estudos.

Quadro 1 - Características dos estudos incluídos na revisão integrativa

(continua)

\begin{tabular}{|l|c|l|l|}
\hline $\begin{array}{l}\text { Autores } \\
\text { Tipo de estudo } \\
\text { Evidência }\end{array}$ & $\begin{array}{l}\text { Ano } \\
\text { País }\end{array}$ & \multicolumn{1}{|c|}{ Objetivo } & \multicolumn{1}{c|}{ Resultados } \\
\hline $\begin{array}{l}\text { Barros APZ, } \\
\text { Lipinski JM, } \\
\begin{array}{l}\text { Sehnem GD, } \\
\text { Rodrigues AN, } \\
\text { Zambiazi ES }\end{array}\end{array}$ & 2017 & $\begin{array}{l}\text { Identificar o } \\
\text { conhecimento dos } \\
\text { enfermeiros que } \\
\text { realizaram o pré-natal, } \\
\text { acerca do PP e sua } \\
\text { importância }\end{array}$ & $\begin{array}{l}\text { Os entrevistados tinham a concepção } \\
\text { de que o PP possibilitava às gestantes } \\
\text { terem conhecimento sobre o direito } \\
\text { do acompanhante, tipo de luz e som } \\
\text { no ambiente de parto, ingesta livre } \\
\text { de líquidos, uso de analgesia ou } \\
\text { Estudótodos qualitativo farmacológicos para } \\
\text { alívio da dor, posição de parir, e } \\
\text { outras condutas que influenciariam o } \\
\text { desfecho positivo do parto. }\end{array}$ \\
\hline VI
\end{tabular}




\begin{tabular}{|c|c|c|c|}
\hline $\begin{array}{l}\text { Autores } \\
\text { Tipo de estudo } \\
\text { Evidência }\end{array}$ & $\begin{array}{l}\text { Ano } \\
\text { País }\end{array}$ & Objetivo & Resultados \\
\hline $\begin{array}{l}\text { Cortés MS, } \\
\text { Barranco DA, } \\
\text { Jordana MC, } \\
\text { Roche MEM }{ }^{(5)} \\
\text { Estudo de coorte } \\
\text { IV }\end{array}$ & $\begin{array}{l}2015 \\
\text { Espanha }\end{array}$ & $\begin{array}{l}\text { Conhecer, analisar e } \\
\text { descrever a situação } \\
\text { atual dos planos de parto, } \\
\text { comparando o processo } \\
\text { de parto e sua finalização } \\
\text { entre as mulheres que } \\
\text { apresentaram e as que } \\
\text { não apresentaram um PP. }\end{array}$ & $\begin{array}{l}\text { O uso do PP proporcionou aumento } \\
\text { das boas práticas obstétricas, como } \\
\text { o contato pele a pele, escolha na } \\
\text { posição de parir, ingestão livre de } \\
\text { líquidos, clampeamento tardio do } \\
\text { cordão umbilical e aumento no } \\
\text { número de partos normais. }\end{array}$ \\
\hline $\begin{array}{l}\text { Biescas H, Benet M, } \\
\text { Pueyo MJ, Rubio A, } \\
\text { Pla M, Pérez BM, } \\
\text { et al }^{(6)} \\
\text { Estudo qualitativo } \\
\text { VI }\end{array}$ & $\begin{array}{r}2017 \\
\text { Espanha }\end{array}$ & $\begin{array}{l}\text { Descrever e analisar } \\
\text { os planos de parto } \\
\text { produzidos pelos } \\
\text { hospitais participantes, } \\
\text { a fim de reunir } \\
\text { conhecimento sobre as } \\
\text { opções disponíveis para } \\
\text { as mulheres. }\end{array}$ & $\begin{array}{l}\text { A maioria dos PP incluiu a opção } \\
\text { de participação ativa do parceiro } \\
\text { no parto; informações acerca do } \\
\text { espaço físico; direito a privacidade; } \\
\text { opção de beber líquidos durante o } \\
\text { parto; mencionou monitoramento } \\
\text { fetal; uso ou não de ocitocina. Todos } \\
\text { abordaram métodos para alívio da } \\
\text { dor e mobilidade livre. }\end{array}$ \\
\hline $\begin{array}{l}\text { Mouta RJO, Silva TMA, } \\
\text { Melo PTS, Lopes NS, } \\
\text { Moreira VA } \\
\text { Estudo de caso } \\
\text { IV }\end{array}$ & $\begin{array}{l}2017 \\
\text { Brasil }\end{array}$ & $\begin{array}{l}\text { Analisar como o } \\
\text { PP propiciou o } \\
\text { empoderamento } \\
\text { feminino durante o } \\
\text { trabalho de parto e } \\
\text { parto. }\end{array}$ & $\begin{array}{l}\text { A aplicação do PP propiciou o } \\
\text { empoderamento e autonomia das } \\
\text { gestantes. Além disso, permitiu que } \\
\text { elas se sentissem mais humanas e } \\
\text { respeitadas. }\end{array}$ \\
\hline $\begin{array}{l}\text { Aragon M, Chhoa E, } \\
\text { Dayan R, } \\
\text { Kluftinger A, } \\
\text { Lohn Z, Buhler } \mathrm{K}^{(12)} \\
\text { Estudo transversal } \\
\text { IV }\end{array}$ & 2013 & $\begin{array}{l}\text { Compreender as } \\
\text { perspectivas das } \\
\text { mulheres e profissionais } \\
\text { da assistência e apoiar } \\
\text { as pessoas no uso de } \\
\text { planos de parto. }\end{array}$ & $\begin{array}{l}\text { Compreensão das preferências } \\
\text { e desejos da mulher durante o } \\
\text { parto; é útil para fins educativos; } \\
\text { proporciona autonomia para as } \\
\text { gestantes; torna o parto mais positivo } \\
\text { e aumenta a sensação de controle e } \\
\text { empoderamento das mulheres. }\end{array}$ \\
\hline $\begin{array}{l}\text { Magoma M, } \\
\text { Requejo J, } \\
\text { Campbell O, } \\
\text { Lousens S, } \\
\text { Merialdi M, } \\
\text { Filippi } V^{(13)} \\
\text { Ensaio clínico } \\
\text { randomizado } \\
\text { II }\end{array}$ & $\begin{array}{l}2013 \\
\text { África }\end{array}$ & $\begin{array}{l}\text { Determinar a eficácia } \\
\text { dos planos de parto no } \\
\text { estímulo à busca por } \\
\text { cuidados especializados } \\
\text { no parto e pós-parto } \\
\text { em um distrito rural no } \\
\text { norte da Tanzânia. }\end{array}$ & $\begin{array}{l}\text { A introdução do PP no pré-natal } \\
\text { aumentou a busca pelas unidades de } \\
\text { saúde. }\end{array}$ \\
\hline $\begin{array}{l}\text { Anderson CM, } \\
\text { Monardo R, } \\
\text { Soon R, Lum J, } \\
\text { Tschann M, } \\
\text { Kaneshiro B } \\
\text { Estudo de coorte } \\
\text { IV }\end{array}$ & $\begin{array}{l}2017 \\
\text { EUA }\end{array}$ & $\begin{array}{l}\text { Descrever os escores de } \\
\text { comunicação, confiança } \\
\text { e satisfação após o } \\
\text { parto em um grupo de } \\
\text { pacientes que utilizaram } \\
\text { um PP padronizado. }\end{array}$ & $\begin{array}{l}\text { Análises descritivas mostraram } \\
\text { aumento nos escores de comunicação } \\
\text { (média=8.7), confiança (média=8.8) e } \\
\text { satisfação }(\text { média=9.2). }\end{array}$ \\
\hline
\end{tabular}


Quadro 1 - Características dos estudos incluídos na revisão integrativa

\begin{tabular}{|c|c|c|c|}
\hline $\begin{array}{l}\text { Autores } \\
\text { Tipo de estudo } \\
\text { Evidência }\end{array}$ & $\begin{array}{l}\text { Ano } \\
\text { País }\end{array}$ & Objetivo & Resultados \\
\hline $\begin{array}{l}\text { Gomes RPC, } \\
\text { Silva RDS, } \\
\text { Oliveira DCCD, } \\
\text { Manzo BF, } \\
\text { Guimarães GDL, } \\
\text { Souza KVD }^{(15)} \\
\text { Estudo } \\
\text { quantitativo } \\
\text { descritivo } \\
\text { VI }\end{array}$ & $\begin{array}{l}2017 \\
\text { Brasil }\end{array}$ & $\begin{array}{l}\text { Caracterizar os desejos e } \\
\text { expectativas de gestantes } \\
\text { descritos em um PP. }\end{array}$ & $\begin{array}{l}\text { Todas as mulheres desejavam ser } \\
\text { acompanhadas, indicando o marido } \\
\text { como acompanhante. Desejavam } \\
\text { ingerir suco durante o parto, } \\
\text { solicitaram pouca luz, ouvir música, } \\
\text { gostariam de usar chuveiro/banheira, } \\
\text { massagem, relaxamento com a bola } \\
\text { ou banco e analgesia local. }\end{array}$ \\
\hline $\begin{array}{l}\text { Hidalgo-Loperoza P, } \\
\text { Hidalgo-Maestre M, } \\
\text { Rodríguez-Borrego A }^{(16)} \\
\text { Estudo } \\
\text { quantitativo } \\
\text { descritivo } \\
\text { IV }\end{array}$ & $\begin{array}{r}2017 \\
\text { Espanha }\end{array}$ & $\begin{array}{l}\text { Conhecer o grau de } \\
\text { cumprimento das } \\
\text { solicitações que as } \\
\text { mulheres registraram } \\
\text { em seus planos de } \\
\text { parto e determinar sua } \\
\text { influência nos principais } \\
\text { resultados obstétricos e } \\
\text { neonatais. }\end{array}$ & $\begin{array}{l}\text { Identificou-se que os recém-nascidos } \\
\text { de mães com alto cumprimento do } \\
\text { plano apresentaram pontos maiores } \\
\text { nos escores de apgar no primeiro } \\
\text { minuto e melhores pontuações no } \\
\text { ph do cordão umbilical do que } \\
\text { em crianças de mães com baixo } \\
\text { cumprimento. }\end{array}$ \\
\hline $\begin{array}{l}\text { Silva ALNV, Neves } \\
\text { AB, Sgarbi AKG, } \\
\text { Souza RA }{ }^{(17)} \\
\text { Revisão } \\
\text { Sistemática } \\
\text { I }\end{array}$ & $\begin{array}{l}2017 \\
\text { Brasil }\end{array}$ & $\begin{array}{l}\text { Refletir através dos } \\
\text { estudos sobre a } \\
\text { importância do plano } \\
\text { de parto para o } \\
\text { empoderamento da } \\
\text { mulher no pré-natal, } \\
\text { parto e puerpério. }\end{array}$ & $\begin{array}{l}\text { O plano de parto é uma ferramenta } \\
\text { primordial para estimular o } \\
\text { empoderamento das gestantes e torna } \\
\text { a mulher protagonista de seu próprio } \\
\text { parto. É um instrumento educativo } \\
\text { de alto potencial, que estimula a } \\
\text { comunicação. }\end{array}$ \\
\hline $\begin{array}{l}\text { Silva JC, Rodrigues } \\
\text { MS }^{(18)} \\
\text { Estudo descritivo } \\
\text { qualitativo } \\
\text { VI }\end{array}$ & $\begin{array}{l}2017 \\
\text { Brasil }\end{array}$ & $\begin{array}{l}\text { Verificar como o PP } \\
\text { pode influenciar na } \\
\text { realização das boas } \\
\text { práticas na assistência } \\
\text { ao parto de puérperas } \\
\text { que construíram o PP } \\
\text { em uma Unidade Básica } \\
\text { de Saúde de Sete Lagos, } \\
\text { MG. }\end{array}$ & $\begin{array}{l}\text { Foi verificado que, ao utilizar as } \\
\text { práticas expressadas no PP, as } \\
\text { mulheres relataram mudança } \\
\text { na assistência obstétrica, o que } \\
\text { proporcionou maior satisfação com } \\
\text { o parto e a equipe de saúde. Teve } \\
\text { impacto também no fortalecimento } \\
\text { de autonomia e empoderamento } \\
\text { feminino. }\end{array}$ \\
\hline $\begin{array}{l}\text { Méndez FSF, } \\
\text { Ferrer LTV, Muñoz } \\
\text { MMN, Gómez JAR, } \\
\text { Jaime AA }{ }^{(19)} \\
\text { Estudo Transversal } \\
\text { IV }\end{array}$ & $\begin{array}{c}2018 \\
\text { Espanha }\end{array}$ & $\begin{array}{l}\text { Determinar o nível de } \\
\text { satisfação das puérperas } \\
\text { em relação ao seu PP. }\end{array}$ & $\begin{array}{l}\text { A maioria das mulheres com PP } \\
\text { atingiram suas expectativas no parto } \\
\text { e expressaram melhor satisfação. }\end{array}$ \\
\hline
\end{tabular}

Fonte: Elaboração própria. 
As principais contribuições do PP para O cuidado no período do parto e nascimento, extraídas dos artigos analisados, podem ser assim sintetizadas:

a) permite maior autonomia e liberdade de escolhas das mulheres ${ }^{(6)}$;

b) possibilita a participação ativa das mulheres em seu próprio parto ${ }^{(6)}$;

c) colabora com o desenvolvimento favorável do trabalho de parto $^{(7)}$;

d) facilitador de decisões informadas ${ }^{(12)}$;

e) estimula atendimento de saúde qualificado no período pós-parto e autocuidado ${ }^{(13)}$;

f) ferramenta de comunicação entre equipe de saúde e usuáriaia ${ }^{(14)}$;

g) facilitador de confiança e satisfação da usuária $^{(14)}$;

h) possibilita realizar escolhas informadas ${ }^{(15)}$;

i) contribui para um atendimento qualificado e humanizado ${ }^{(15)}$;

j) permite maior satisfação com o parto ${ }^{(16)}$;

k) colabora para melhores resultados materno e neonatais ${ }^{(16)}$;

1) colabora para melhor qualidade da assistência ao binômio mãe-filho ${ }^{(17)}$.

\section{Discussão}

Com base nos resultados desta revisão, observou-se que o PP deve ser uma ferramenta fundamentada em evidências científicas, para estimular a promoção das boas práticas obstétricas, de modo a estabelecer condutas flexíveis no atendimento à parturiente, garantindo o respeito por suas decisões, vontades e individualidades. Salienta-se que a maioria dos artigos analisados mostrou que a aplicação do PP foi benéfica na prática clínica obstétrica, tornando o trabalho de parto um evento mais humanizado e uma experiência positiva.

Revelou-se que existe uma relação favorável entre o PP e a promoção do nascimento saudável $^{(3-4)}$. Assim, a construção do PP auxilia no estímulo do contato pele a pele da mãe com o bebê após o parto, clampeamento tardio do cordão umbilical e um parto mais natural, o que, de certa forma, diminui as hospitalizações e os gastos desnecessários com a saúde ${ }^{(16-17)}$. A apresentação do PP não influencia apenas na redução de intervenções desnecessárias, mas também contribui para tornar as mulheres mais preparadas e conscientes de seu processo de parto. Além disso, possibilita maior participação, que, por sua vez, desempenha um papel fundamental na redução da ansiedade, do medo e de estresse durante o parto ${ }^{(16)}$.

Nesse mesmo contexto, percebe-se que a introdução do PP durante o pré-natal ocasiona maior satisfação nas mulheres com a consulta e, dessa forma, aumenta o interesse e a busca por cuidados especializados em unidades de saúde durante a gestação e após o parto. Da mesma forma, a aplicação do PP desde o pré-natal permite que os profissionais da assistência compreendam as necessidades individuais de cada mulher atendida ${ }^{(13)}$. Sua implementação garante o respeito ao princípio bioético da autonomia, a melhoria na assistência prestada e a participação ativa da mulher durante esse período, já que as condutas realizadas no parto serão baseadas com respeito às suas escolhas ${ }^{(17)}$.

A construção do PP funciona como uma estratégia de empoderamento feminino, visto que permite autonomia e liberdade para as gestantes planejarem seu próprio parto e, dessa forma, sentirem-se mais confiantes e menos ansiosas. Além disso, proporciona autoconhecimento e entendimento sobre o direito e a necessidade de um parto humanizado, sem intervenções desnecessárias, constituindo-se em uma ferramenta potencializadora do cuidado ${ }^{(1,12,17-18)}$.

No que diz respeito ao parto, o PP proporciona às gestantes a possibilidade da escolha da melhor posição para parir, liberdade de optar pela ingestão de alimentos e líquidos, como também escolha de métodos não farmacológicos de alívio da dor. Tais condutas contribuem de forma benéfica para um parto saudável e, concomitantemente, aumentam a satisfação com o trabalho de parto e nascimento ${ }^{(3-4,12,15,17-18)}$.

Outro ponto positivo, observado nos estudos $^{(12,14)}$, foi o fato de o PP estimular o diálogo entre as gestantes e os profissionais da assistência, 
além de promover a discussão sobre as vontades, desejos e preocupações relacionadas ao parto, uma estratégia conveniente, que contribui para o fortalecimento da confiança entre eles e proporciona maior segurança, já que as práticas realizadas serão de acordo com o documento. Diante disso, o ato de elaborar um PP também funciona como um processo educacional.

No que se refere ao nível de satisfação das gestantes com o parto, observou-se, nos estudos analisados, que essa variável está diretamente associada ao cumprimento do PP. Quanto maior a sua aplicação, melhores são os resultados maternos e neonatais; além disso, a experiência com o parto torna-se mais positiva ${ }^{(16)}$. A insatisfação feminina foi percebida quando as condutas expressas no documento não foram cumpridas durante a assistência. Dessa forma, as expectativas criadas foram vistas pelas gestantes como algo incomum e distantes da realidade ${ }^{(12,19)}$.

Quanto ao grau de conhecimento sobre o PP, é evidente que ainda existe certo desconhecimento tanto das gestantes ${ }^{(7,14)}$ quanto dos profissionais de saúde ${ }^{(8)}$. A maioria das mulheres envolvidas nas pesquisas nunca havia preenchido um PP antes, ou sequer ouviram falar sobre o documento. Essa realidade mostra que o PP ainda é pouco estimulado na prática clínica obstétrica hospitalar e na atenção primária de saúde. Essa problemática foi discutida por vários autores, e tais estudos devem ser levados em consideração, para que haja mudanças no cuidar.

Diante disso, pode-se afirmar que a construção e a implementação do PP representam uma estratégia de grande importância para o processo de humanização e promoção da saúde nas práticas da obstetrícia e neonatologia. Esse instrumento deve ser mais trabalhado, divulgado e estimulado, para que as mulheres apoderem-se desse conhecimento e sintam total autonomia para lidar com o momento do parto.

Destaca-se que há uma produção reduzida de pesquisa de enfermagem que envolva diretamente a temática do PP no Brasil. Acredita-se que, dentre outros fatores, a baixa produção de artigos com forte nível de evidência possa ser justificada pelo fato de se tratar de tema ainda recente e uma prática não consolidada no país. Evidencia-se a necessidade de novas pesquisas que abordem a importância dessa temática para o fortalecimento das boas práticas no parto e nascimento no contexto brasileiro. Entretanto, a maior limitação do estudo pode estar relacionada ao fato de terem sido utilizados na busca somente os Descritores em Ciências da Saúde (DeCS e MeSH) para recuperar os artigos nas bases de dados eletrônicas, pois se constatou que PP é um termo ainda não indexado, o que pode ter contribuído para que alguns trabalhos não tenham sido acessados.

\section{Conclusão}

Evidenciou-se que o PP é uma ferramenta efetiva que favorece o empoderamento e a autonomia feminina, além disso, promove maior satisfação com o parto, melhor comunicação com os profissionais envolvidos e resultados maternos/neonatais mais satisfatórios. Estimular a construção do PP em unidades primárias de saúde e o seu grau de cumprimento em maternidades é algo fundamental para favorecer tais resultados. Portanto, torna-se necessário a adesão do instrumento pelos serviços de saúde, para que o PP seja um instrumento de excelência e de qualidade para o cuidado materno infantil.

Espera-se que este estudo possa estimular discussões acerca do tema, e que novas pesquisas sejam realizadas para fomentar a implantação desse documento na assistência obstétrica brasileira. Para que o PP seja um instrumento de excelência e de qualidade para o cuidado materno infantil, torna-se necessária a sua adesão pelos serviços de saúde.

\section{Colaborações:}

1 - concepção, projeto, análise e interpretação dos dados: Wanessa Nathally de Santana Silva, Joicy Amorim Francisco de Azevêdo, Viviane Rolim de Holanda, Ana Lisa do Vale Gomes e Geyslane Pereira Melo de Albuquerque;

2 - redação do artigo e revisão crítica relevante do conteúdo intelectual: Wanessa 
Nathally de Santana Silva, Joicy Amorim Francisco de Azevêdo, Viviane Rolim de Holanda, Ana Lisa do Vale Gomes e Geyslane Pereira Melo de Albuquerque;

3 - aprovação final da versão a ser publicada: Wanessa Nathally de Santana Silva, Joicy Amorim Francisco de Azevêdo, Viviane Rolim de Holanda, Ana Lisa do Vale Gomes e Geyslane Pereira Melo de Albuquerque.

\section{Referências}

1. Leal MC, Pereira APE, Domingues RMSM, Filha MMT, Dias MAB, Pereira MN, et al. Intervenções obstétricas durante o trabalho de parto e parto em mulheres brasileiras de risco habitual. Cad Saúde Pública [Internet]. 2014 [cited 2018 May 23];30(Suppl 1):S17-S32. Available from: http://www.scielo.br/ scielo.php?script=sci_arttext\&pid=S0102-311X2014 $001300005 \& \operatorname{lng}=\mathrm{en} \& \mathrm{nrm}=\mathrm{iso} \& \ln \mathrm{l}=\mathrm{pt}$

2. Barros APZ, Lipinski JM, Sehnem GD, Rodrigues AN, Zambiazi ES. Conhecimento de enfermeiras sobre plano de parto. Rev enferm UFSM [Internet]. 2017 [cited 2018 Jun 2];7(1):69-79. Available from: https://periodicos.ufsm.br/reufsm/article/ view $/ 23270$

3. Santos RAA, Melo MCP, Cruz DD. Trajetória de humanização do parto no Brasil a partir de uma revisão integrativa de literatura. Cad Cult Ciênc [Internet]. 2015 [cited 2018 May 18];13(2):77-89. Available from: http://periodicos.urca.br/ojs/ index.php/cadernos/article/view/838

4. Frutuoso LD, Brüggemann OM, Monticelli M, Oliveira ME, Costa R. Percepções do acompanhante de escolha da mulher acerca da organização e ambiência do centro obstétrico. Rev Fund Care Online [Internet]. 2017 [cited 2018 May18];9(2):36370. Available from: http://www.seer.unirio.br/ índex.php/cuidadofundamental/article/view/4928

5. Cortés MS, Barranco DA, Jordana MC, Roche MEM. Uso e influência dos Planos de Parto e Nascimento no processo de parto humanizado. Rev Latino-Am Enfermagem [Internet]. 2015 [cited 2018 Jun 8];23(3):520-6. Available from: http://www.redalyc. org/articulo.oa?id=281442223021

6. Biescas H, Benet M, Pueyo MJ, Rubio A, Pla M, Pérez BM, et al. A critical review of the birth plan use in Catalonia. Sex Reprod Healthc [Internet]. 2017 [cited 2018 May 8];13:41-50. Available from: http://clok.uclan.ac.uk/19880/
7. Mouta RJO, Silva TMA, Melo PTS, Lopes NS, Moreira VA. Plano de parto como estratégia de empoderamento feminino. Rev baiana enferm [Internet]. 2017 [cited 2018 Jun 10];31(4):e20275. Available from: https:/portalseer.ufba.br/index.php/ enfermagem/article/view/20275

8. Ministério Público de Pernambuco. Procuradoria Geral de Justiça. Humanização do parto. Nasce o respeito [Internet]. Recife; 2015 [cited 2018 Sep 4]. Available from: http://www.mppe.mp.br/ mppe/attachments/article/4240/cartilha\%20 humanizacao\%20do\%20parto\%20pdf.pdf

9. Souza MT, Silva MD, Carvalho R. Revisão integrativa: o que é e como fazer. Einstein [Internet]. 2010 [cited 2018 Jun 10];8(1):102-6. Available from: http://www.scielo.br/pdf/eins/ v8n1/pt_1679-4508-eins-8-1-0102

10. Ursi ES, Gavão CM. Prevenção de lesões de pele no perioperatório: revisão integrativa da literatura. Rev Latino-Am Enfermagem [Internet]. 2006 [cited 2018 Nov 10];14(1):124-31. Available from: http://www. scielo.br/pdf/rlae/v14n1/v14n1a17.pdf

11. Melnyk BM, Fineout-Overholt E. Making the case for evidence-based practice and cultivating a spirit of inquiry. In: Melnyk BM, Fineout-Overholt E. Evidence-based practice in nursing \& healthcare. A guide to best practice. $2 \mathrm{a}$ ed. Philadelphia: Wolters Kluwer, Lippincott Willians \& Wilkins; 2011. p. 3-24.

12. Aragon M, Chhoa E, Dayan R, Kluftinger A, Lohn Z, Buhler K. Perspectives of expectant women and health care providers on birth plans. J Obstet Gynaecol Can [Internet]. 2013 [cited 2018 Jun 10];35(11):979-85. Available from: https:// www.ncbi.nlm.nih.gov/pubmed/24246397

13. Magoma M, Requejo J, Campbell O, Cousens S, Merialdi M, Filippi V. The effectiveness of birth plans in increasing use of skilled care at delivery and postnatal care in rural Tanzania: a cluster randomised trial. Trop Med Int Health [Internet]. 2013 [cited 2018 May 20];18(4):435-43. Available from: https://www.ncbi.nlm.nih.gov/ pubmed/23383733

14. Anderson CM, Monardo R, Soon R, Lum J, Tschann M, Kaneshiro B. Patient Communication, Satisfaction, and Trust Before and After Use of a Standardized Birth Plan. Hawai'i J Med Public Health [Internet]. 2017 [cited 2018 May 20];76(11):305-9. Available from: https://www. ncbi.nlm.nih.gov/pubmed/29164014 
15. Gomes RPC, Silva RDS, Oliveira DCCD, Manzo BF, Guimarães GDL, Souza KVD. Plano de parto em rodas de conversa: escolhas das mulheres. Reme - Rev Min Enferm [Internet]. 2017 [cited Jul 6];21:e1033. Available from: http://www.reme.org. br/artigo/detalhes/1169

16. Hidalgo-Lopezosa P, Hidaldo-Maestre M, Rodríguez-Borrego MA. O cumprimento do plano de parto e sua relação com os resultados maternos e neonatais. Rev Latino-Am Enfermagem [Internet]. 2017 [cited 2018 Aug 10];25:e2953. Available from: http://www.scielo.br/scielo.php?pid=S0104$11692017000100399 \&$ script=sci_arttext\&tlng=PT

17. Silva ALNV, Neves AB, Sgarbi AKG, Souza RA. Plano de parto: ferramenta para o empoderamento de mulheres durante a assistência de enfermagem. Rev enferm UFSM [Internet]. 2017 [cited 2018 Aug
10];7(1):144-51. Available from: https://periodicos. ufsm.br/reufsm/article/view/22531

18. Silva JC, Rodrigues MS. Boas práticas na assistência ao parto: implicações do plano de parto. Rev Bras Ciênc Vida [Internet]. 2017 [cited 2018 Oct 23];5(4):1-27. Available from: http:// jornal.faculdadecienciasdavida.com.br/index.php/ RBCV/article/view/403

19. Méndez FSF, Ferrer LTV, Muñoz MMN, Gómez JAR, Jaime AA. El empoderamiento de la gestante y su satisfacción durante el parto. Dilemata [Internet]. 2018 [cited 2018 Dec 2];(26):207-15. Available from: https://www.dilemata.net/revista/index.php/ dilemata/article/view/412000189

Recebido: 5 de agosto de 2019 Aprovado: 17 de outubro de 2019

Publicado: 17 de março de 2020

A Revista Baiana de Enfermagem utiliza a Licença Creative Commons - Atribuição-NãoComercial 4.0 Internacional.

https://creativecommons.org/licenses/by-nc/4.0/ Este artigo é de acesso aberto distribuído sob os termos da Licença Creative Commons (CC BY-NC). Esta licença permite que outros remixem, adaptem e criem a partir do seu trabalho para fins não comerciais. Embora os novos trabalhos tenham de lhe atribuir o devido crédito e não possam ser usados para fins comerciais, os usuários não têm de licenciar esses trabalhos derivados sob os mesmos termos. 